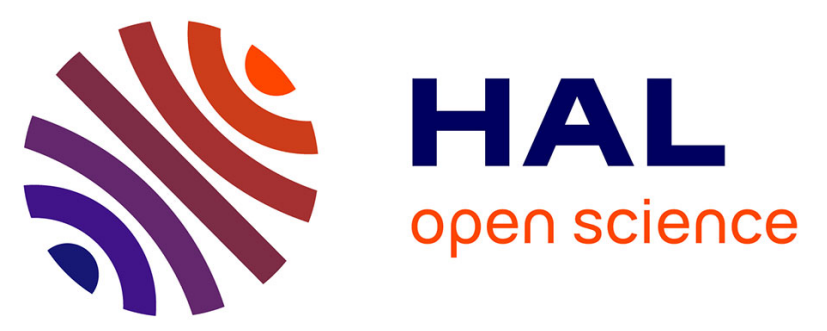

\title{
Enregistrement fluviatile et paléoenvironnements au Pléistocène supérieur sur la bordure méridionale atlantique de l'Anti-Atlas (Oued Assaka, S-O marocain)
}

Luc Wengler, André Weisrock, Jacques-Élie Brochier, Jean Philippe Brugal,

Michel Fontugne, Frédéric Magnin, Julien Mathieu, Norbert Mercier, Abderrahman Ouammou, Jean-Louis Reyss, et al.

\section{To cite this version:}

Luc Wengler, André Weisrock, Jacques-Élie Brochier, Jean Philippe Brugal, Michel Fontugne, et al.. Enregistrement fluviatile et paléoenvironnements au Pléistocène supérieur sur la bordure méridionale atlantique de l'Anti-Atlas (Oued Assaka, S-O marocain). Quaternaire, 2002, 13 (3), pp.179-192. 10.3406/quate.2002.1710 . hal-02497453

\section{HAL Id: hal-02497453 \\ https://hal.science/hal-02497453}

Submitted on 14 Oct 2020

HAL is a multi-disciplinary open access archive for the deposit and dissemination of scientific research documents, whether they are published or not. The documents may come from teaching and research institutions in France or abroad, or from public or private research centers.
L'archive ouverte pluridisciplinaire HAL, est destinée au dépôt et à la diffusion de documents scientifiques de niveau recherche, publiés ou non, émanant des établissements d'enseignement et de recherche français ou étrangers, des laboratoires publics ou privés.

\section{(1)(1) $\$(0)$}

Distributed under a Creative Commons Attribution - NonCommercial - ShareAlikel 4.0 


\section{Enregistrement fluviatile et paléoenvironnements au Pléistocène} supérieur sur la bordure méridionale atlantique de l'Anti-Atlas (Oued Assaka, S-O marocain)

Luc Wengler, André Weisrock, Jacques-Élie Brochier-do not use, Jean Philippe Brugal, Michel Fontugne, Frédéric Magnin, Julien Mathieu, Norbert Mercier, Abderrahman Ouammou, Jean-Louis Reyss, Franck Sénégas, Hélène Valladas, Laurent Wahl

\section{Citer ce document / Cite this document :}

Wengler Luc, Weisrock André, Brochier-do not use Jacques-Élie, Brugal Jean Philippe, Fontugne Michel, Magnin Frédéric, Mathieu Julien, Mercier Norbert, Ouammou Abderrahman, Reyss Jean-Louis, Sénégas Franck, Valladas Hélène, Wahl Laurent. Enregistrement fluviatile et paléoenvironnements au Pléistocène supérieur sur la bordure méridionale atlantique de I'Anti-Atlas (Oued Assaka, S-O marocain). In: Quaternaire, vol. 13, n³-4, 2002. Événements rapides, instabilités, changements culturels au Quaternaire. pp. 179-192;

doi : https://doi.org/10.3406/quate.2002.1710

https://www.persee.fr/doc/quate_1142-2904_2002_num_13_3_1710

Fichier pdf généré le 19/04/2018 


\title{
Résumé
}

L'oued Assaka draîne un important bassin versant sur la bordure sud de l'Anti-Atlas occidental et ses marges sahariennes dans la région de Guelmim. II traverse les derniers chaînons anti-atlasiques plissés avant de déboucher dans l'océan Atlantique. Dans cette partie de son cours, il a édifié un système de terrasses alluviales étagées pour les plus anciennes et emboîtées pour les dernières. L'une d'entre elles est particulièrement remarquable à la fois par sa puissance, la variété de ses dépôts, ainsi que par leur contenu. Les divers faciès sédimentaires visibles grâce à une forte incision linéaire actuelle qui recoupe l'ensemble de la formation permettent de reconstituer les paléoenvironnements, la dynamique passée de l'oued et de proposer une interprétation des dépôts en relation avec l'évolution climatique régionale et globale. Cette séquence sédimentaire paraît avoir enregistré en haute résolution des événements climatiques rapides durant les stades isotopiques 5 à 2. Elle fournit des repères concernant la réponse du continent africain face aux changements climatiques globaux à la frontière des domaines méditerranéen et saharien.

\begin{abstract}
The Wadi Assaka drains a large watershed on the southwest border of Anti-Atlas and its Saharan edges in the Guelmim area. It crosses the last folded chains of the Anti- Atlas before flowing into the Atlantic Ocean. In this part of its course, it aggraded a large system of alluvial terraces. The oldest of them are stepped terraces, the last ones are inset terraces. One of them is particularly interesting by its thickness, its various deposits as well as their content. The whole formation is now deeply cut, so the different sedimentary faciès can be observed and it is possible to reconstruct the palaeoenvironments, the ancient dynamics of the river and to connect the deposits with the regional and general climatic evolution. This stratigraphy has seemingly recorded rapid climatic events with a high resolution during the isotopic stages 5 to 2 It gives reference points about the way the African continent responded to global climatic changes between the Mediterranean and Saharan areas.
\end{abstract}




\title{
ENREGISTREMENT FLUVIATILE ET PALÉOENVIRONNEMENTS AU PLÉISTOCENE SUPÉRIEUR SUR LA BORDURE ATLANTIQUE DE L'ANTI-ATLAS (Oued ASSAKA, S-O marocain)
}

\author{
Luc WENGLER', André WEISROCK ${ }^{2}$, Jacques-Elie BROCHIER ${ }^{3}$, \\ Jean-Philip BRUGAL ${ }^{4}$, Michel FONTUGNE ${ }^{s}$, Frédéric MAGNIN', \\ Julien MATHIEU ${ }^{2}$, Norbert MERCIER ${ }^{5}$, Abderrahmane OUAMMOU ${ }^{7}$, \\ Jean-Louis REYSS ${ }^{5}$, Franck SENEGAS ${ }^{8}$, Hélène VALLADAS ${ }^{5}$ et Laurent WAHL ${ }^{2}$
}

\begin{abstract}
RESUME
L'oued Assaka draîne un important bassin versant sur la bordure sud de l'Anti-Atlas occidental et ses marges sahariennes dans la région de Guelmim. Il traverse les derniers chaînons anti-atlasiques plissés avant de déboucher dans l'océan Atlantique. Dans cette partie de son cours, il a édifié un système de terrasses alluviales étagées pour les plus anciennes et emboîtées pour les dernières. L'une d'entre elles est particulièrement remarquable à la fois par sa puissance, la variété de ses dépôts, ainsi que par leur contenu. Les divers faciès sédimentaires visibles grâce à une forte incision linéaire actuelle qui recoupe l'ensemble de la formation permettent de reconstituer les paléoenvironnements, la dynamique passée de l'oued et de proposer une interprétation des dépôts en relation avec l'évolution climatique régionale et globale. Cette séquence sédimentaire paraît avoir enregistré en haute résolution des événements climatiques rapides durant les stades isotopiques 5 à 2 . Elle fournit des repères concernant la réponse du continent africain facc aux changements climatiques globaux à la frontière des domaines méditerranéen et saharien.
\end{abstract}

Mots-clés : S-O Maroc, Pléistocène supérieur, Holocène, terrasse alluviale, stratigraphie, paléoclimatologie, faunes de mammifères, malacofaune, environnement, événements rapides.

\begin{abstract}
UPPER PLEISTOCENE FLUVIAL RECORD AND PALAEOENVIRONMENTS ON THE ATLANTIC BORDER OF ANTI-ATLAS (Wadi Assaka, S-W Morocco)

The Wadi Assaka drains a large watershed on the southwest border of Anti-Atlas and its Saharan edges in the Guelmim area. It crosses the last folded chains of the Anti-Atlas before flowing into the Atlantic Ocean. In this part of its course, it aggraded a large system of alluvial terraces. The oldest of them are stepped terraces, the last ones are inset terraces. One of them is particularly interesting by its thickness, its various deposits as well as their content. The whole formation is now deeply cut, so the different sedimentary facies can be observed and it is possible to reconstruct the palaeoenvironments, the ancient dynamics of the river and to connect the deposits with the regional and general climatic evolution. This stratigraphy has seemingly recorded rapid climatic events with a high resolution during the isotopic stages 5 to 2 . It gives reference points about the way the African continent responded to global climatic changes between the Mediterranean and Saharan areas.
\end{abstract}

Key-words : S.W. Morocco, Upper Pleistocene, Holocene, alluvial terraces, stratigraphy, palaeoclimatology, mammal faunas, malacofauna, environment, rapid events.

\footnotetext{
1 Université de Perpignan. 52. avenue de Villeneuve. F-66860 Perpignan et UMR 5590 du CNRS. Préhistoire. milieux et paléoenvironnements quaternaires. 2 Université de Nancy 2. BP 33-97. F-54015 Nancy Cedex.

3 Laboratoire de Paléontologie humaine et de Préhistoire. UMR 6569 du CNRS. Université de Provence. centre Saint-Charles. Place Victor Hugn, Case 54. F-1.33.3। Marseille Cedex 3.

4 ESEP. UMR 6636 du CNRS, MMSH. BP 647, 5 rue du Château de l'Horloge. F-13094 Aix-en-Provence cedex 2.

5 Laboratoire des Sciences du Climat et de l'Environnement - CEA/CNRS. Avenue de la Terrasse. F-91198 Gif-sur-Yvette Cedex.

6 Faculté des Sciences et Techniques. Centre Saint-Jérôme. IMEP-URA I152 CNRS. c.461. Avenue Normandie-Viemens. 13397 Marseille cedex 20.

7 Université Ibnou Zohr, département de Géographie. Cité Dakhla, Agadir. Maroc.

8 Laboratoire de Paléontologie et EPHE. ISEM cc (064. université de Montpellier II. place E. Bataillon. F-34090, Montpellier Cedex 05.
} 


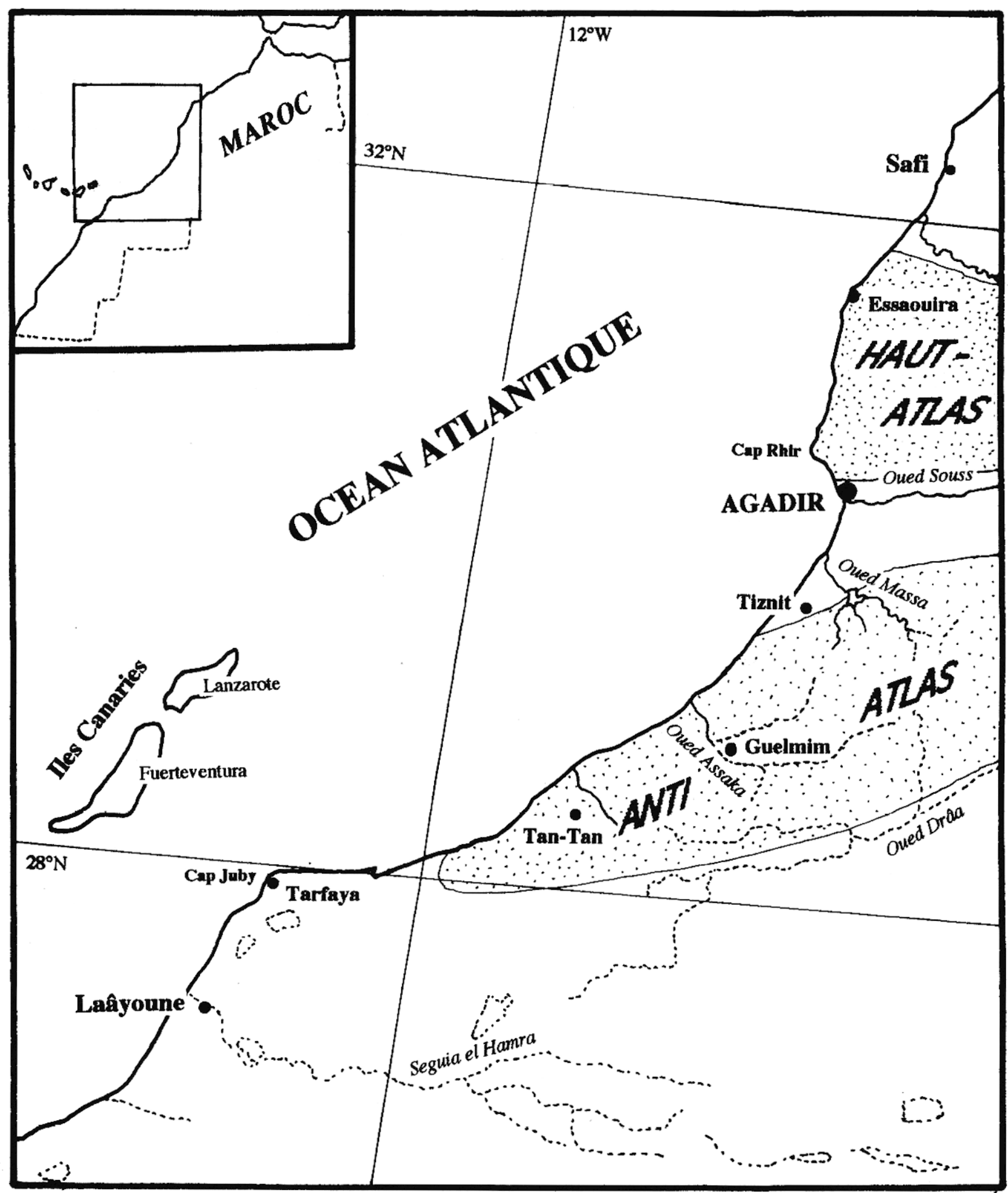

Fig. 1 : Carte de situation de l'oued Assaka.

Fig. 1 : Location map of Wadi Assaka.

\section{1 - INTRODUCTION}

L'oued Assaka ou oued Noun est un fleuve côtier dont l'exutoire est situé à $250 \mathrm{~km}$ au Sud d'Agadir, à la latitude de la ville de Guelmim (fig. 1). Son bassin-versant s'étend sur 6500 à $7000 \mathrm{~km}^{2}$ et se développe sur le versant sud de l'Anti-Atlas qui jouxte le domaine saharien. Les formations géologiques qui l'alimentent en alluvions sont aussi bien granitiques, schisto-gréseuses et quartzitiques que calcaires. Elles correspondent à la partie supérieure du Précambrien (boutonnière d'Ifni) et au Paléozoïque inférieur qui forment une partie de la couverture sédimentaire anti-atlasique. L'érosion y a mis au jour les granites du socle, les intrusions granitiques hercyniennes, ainsi que des complexes volcaniques du même âge, notamment dans la région d'Ifni, qui sont à l'origine d'ignimbrites. L'importance du bassin-versant et la variété des formations que l'on y rencontre font de cet oued un organisme fluviatile différent des autres petits fleuves côtiers. En effet, la présence d'un karst ancien 
développé dans les formations calcaires entretient des sources qui sont à l'origine de l'alimentation pérenne de cet oued, notamment dans la partie inférieure de son cours, alors qu'il traverse, dans la région d'Assaka, les derniers chaînons anti-atlasiques par une série de gorges larges de quelques dizaines à quelques centaines de mètres le conduisant à l'océan Atlantique (Andres, 1977). Cette alimentation entretient un ensemble de gueltas dans les petits bassins intermédiaires, où les animaux peuvent venir s'abreuver même durant les années les plus sèches (1998-2001).

Outre le fait que cet oued a développé un système de terrasses alluviales, son intérêt réside dans la formation principale de ce système, qui atteint plusieurs dizaines de mètres d'épaisseur, ampleur exceptionnelle pour l'Afrique du Nord. Cette formation du Pléistocène supérieur a enregistré certaines variations plus ou moins rapides du milieu et il est possible d'y obtenir des enregistrements à haute résolution. De plus, elle présente l'avantage de contenir des vestiges paléontologiques et archéologiques, et son étude pluridisciplinaire devrait fournir des indications chronologiques et paléoenvironnementales pour reconstituer les milieux et climats de cette période, afin de comprendre comment cette partie de la marge nord-saharienne du continent africain réagit face aux tendances climatiques globales obtenues par l'analyse des carottes glaciaires et marines.

L'étude de la partie distale du cours de l'oued Assaka sera d'abord envisagée sur un plan morphologique, ce qui permet de définir plusieurs niveaux de terrasses alluviales. Chaque niveau correspond en fait à une formation sédimentaire qui peut comporter dans certains cas deux séquences sédimentaires d'âge très différent. Leur étude lithostratigraphique permet de séparer plusieurs unités sédimentaires présentant des faciès de lit mineur et des faciès latéraux. On apportera quelques éléments chronologiques actuellement disponibles permettant de situer ces séquences dans le temps en attendant les résultats des analyses en cours, ainsi que des données paléontolo- giques et sédimentologiques autorisant une première approche des paléoenvironnements et des paléoclimats que l'on comparera aux données globales et à celles acquises dans d'autres régions.

\section{2 - LE SYSTÈME DE TERRASSES ALLUVIALES DE L'OUED ASSAKA}

Dans la partie inférieure de son cours, la vallée de l'oued montre l'étagement et l'emboîtement de plusieurs terrasses alluviales (fig. 2) recoupant le relief appalachien de la couverture anti-atlasique formé de dépressions et de chaînons offrant un paysage de collines ou de petites montagnes selon la résistance des roches à l'érosion.

\section{1 - LA PLUS HAUTE TERRASSE}

Elle domine le lit actuel de 65-70 m en moyenne et forme en rive droite une surface d'aplanissement discontinue bien marquée dans le paysage. Cette terrasse est couverte par une nappe de galets en vrac, très hétérométriques, dont les plus gros éléments, noyés dans une matrice limono-sableuse carbonatée et consolidée de couleur beige, sont de dimensions métriques. La nappe de galets repose directement sur le substratum par un contact de type érosif et son épaisseur visible atteint $6 \mathrm{~m}$. Elle ne représente que la partie inférieure d'une formation beaucoup plus importante actuellement très érodée.

La présence à sa surface d'industries lithiques de différentes périodes et notamment des séries d'artefacts correspondant à des faciès d'atelier de débitage, où l'on trouve des pièces typiques du Paléolithique moyen, ne permet en aucune mesure de préciser pour l'instant son attribution chronologique. On peut seulement affirmer qu'elle est très antérieure au Paléolithique moyen.

Après une très puissante incision linéaire qui a pu se produire en plusieurs étapes, la vallée s'est stabilisée dans sa position actuelle, ouvrant dans les schistes et
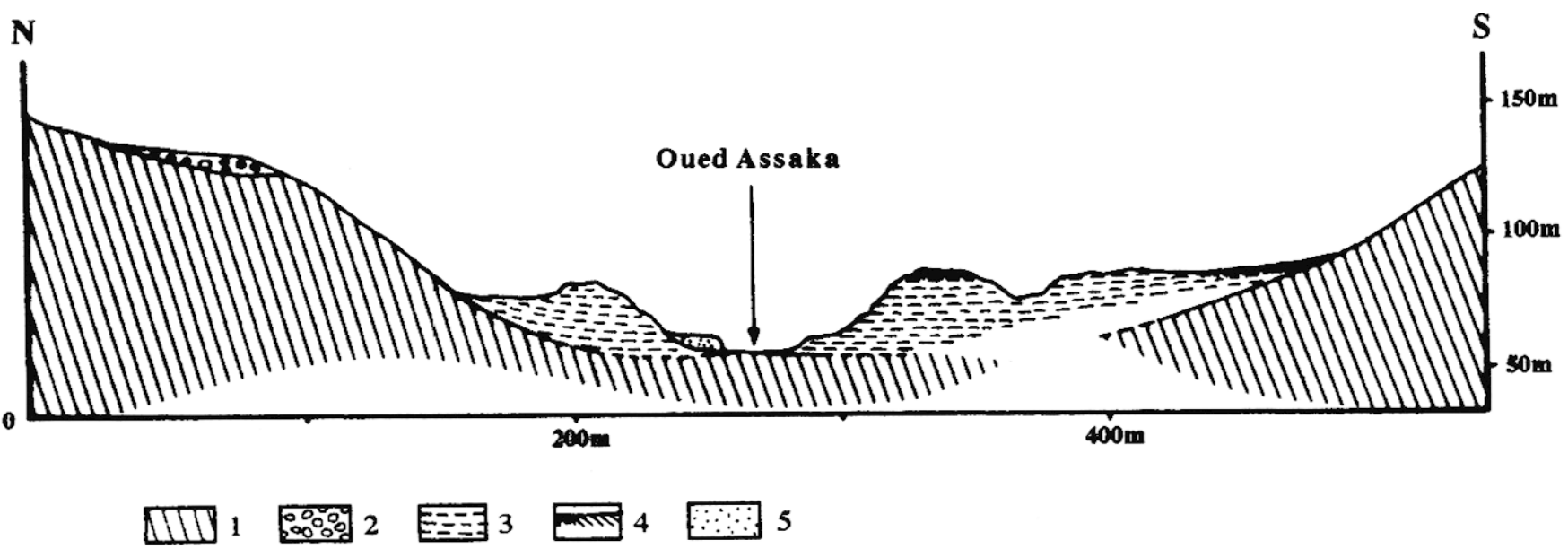

Fig. 2 : Coupe transversale de l'oued Assaka en amont de Bou Jerif et position des terrasses alluviales quaternaires.

$1:$ grès fins verts et silstone cambriens. $2:$ haute terrasse. $3:$ terrasse principale soltanienne $4:$ glacis sommital $.5:$ terrasse holocène.

Fig. 2 : Cross section of Wadi Assaka upstream from Bou Jerif and position of Quaternary alluvial terraces.

1: Cambrian green fine-grained sandstone and siltstone. 2 : high terrace. $3:$ Soltanian main terrace. $4:$ terminal glacis.

5 : Holocene terrace. 

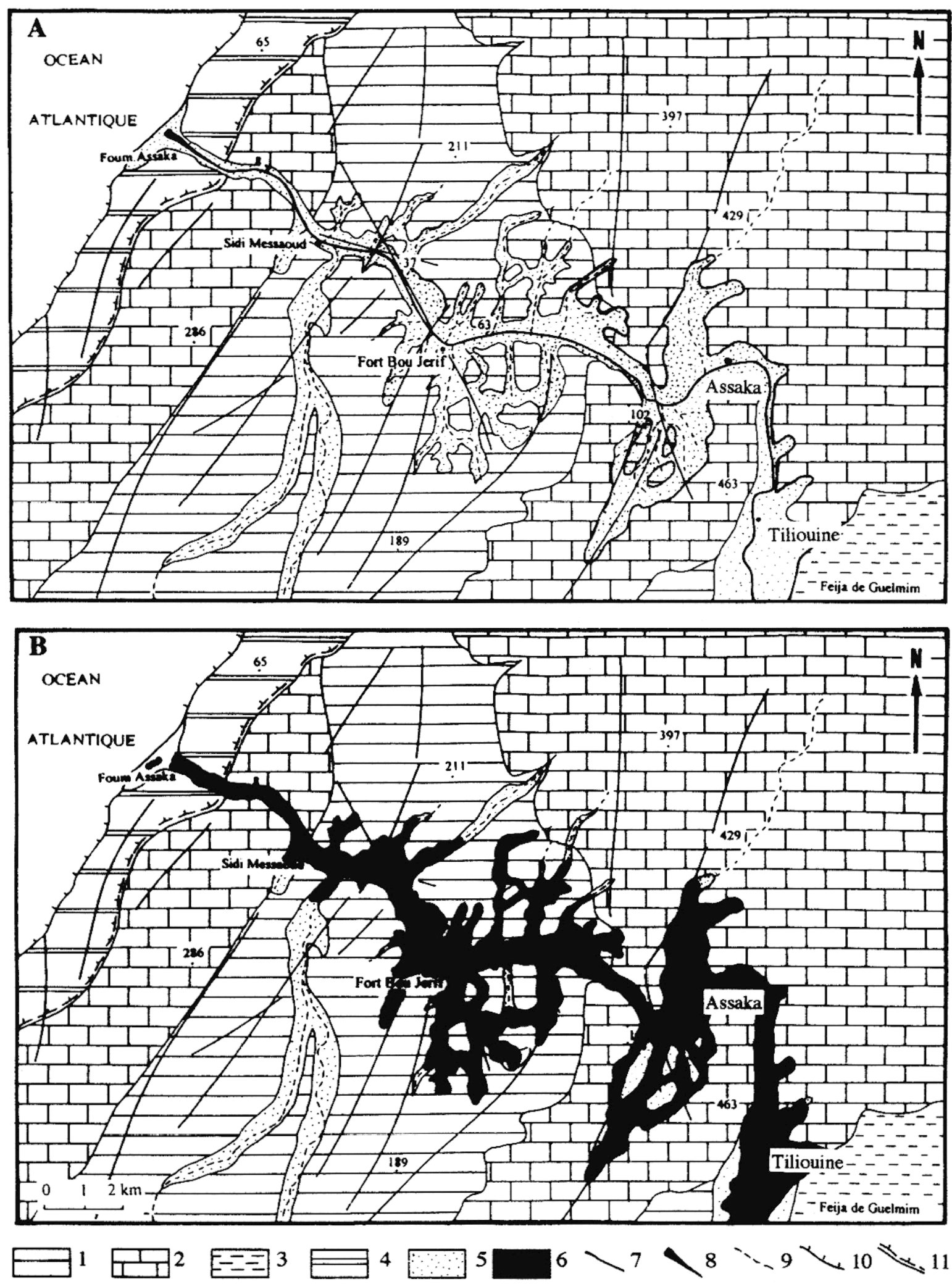

Fig. 3 : A - Lit actuel de l'oued Assaka dans la partie inférieure de son cours. B - Extension maximale de la terrasse soltanienne dans la partie inférieure du cours de l'oued Assaka.

1 : grés fins verts et siltstone argileux cambriens. 2 : calcaires cambriens. $3:$ calcaires lacustres. $4:$ rasa moghrébienne. $5:$ dépôts quaternaires. $6:$ terrasse alluviale soltanienne. $7:$ faille. $8:$ lagune. $9:$ affluent intermittent. $10:$ falaise vive. $11:$ falaise morte.

Fig. 3 : A - Actual river bed in the lower part of Wadi Assaka. B - : Maximum expansion of the soltanian terrace in the lower part of Wadi Assaka.

1: Cambrian green fine-grained sandstone and argillaceous siltstone . $2:$ Cambrian limestone. $3:$ lacustrine limestone. 4 : Moghrebian rasa. $5:$ Quaternary deposits. $6:$ Soltanian alluvial terrace. $7:$ fault. $8:$ lagoon. $9:$ intermittent tributary river. 10 : cliff. 11 : dead cliff. 
quartzites des chaînons anti-atlasiques côtiers une profonde entaille, dont la largeur varie entre 40 et $300 \mathrm{~m}$. C'est dans cette entaille, qui adopte un tracé en baïonnette calqué sur le système de fracture (Oliva, 1974), que l'oued a édifié une accumulation remarquable.

\section{2 - LA TERRASSE PRINCIPALE}

En aval du village d'Assaka, cette formation est omniprésente et constitue le remplissage principal de la vallée. Au maximum de son extension, elle occupait l'ensemble de la vallée et remontait dans les lits d'oueds affluents sur plusieurs centaines de mètres, comme en témoignent certains lambeaux sédimentaires, aujourd'hui disséqués par l'érosion, qui permettent de reconstituer sa paléogéographie (fig. 3). Sa puissance oscille entre 27-28 m à l'amont dans la zone de Fort Bou Jerif et $35 \mathrm{~m}$ plus en aval au niveau du marabout de Sidi Messaoud.

Généralement, dans les oueds maghrébins, la puissance des terrasses alluviales dans leur partie moyenne et distale varie de quelques mètres à plus d'une dizaine de mètres, ces dernières étant plus rares (Weisrock, 1980 ; Ballais, 1981 ; Lefèvre, 1985 ; Barathon, 1986 ; Bhiry et al., 1989 ; Bhiry, 1991 ; Wengler, 1993 ; Zarki, 1999...). Les séquences sédimentaires observées sont dans la majorité des cas directement en relation avec le climat qui gouverne le couple érosion-sédimentation à travers le filtre de la végétation dans les régions tectoniquement peu actives. Ces séquences débutent généralement par une nappe de galets en vrac témoignant d'écoulements torrentiels chenalisés, à laquelle succèdent des dépôts de moindre énergie constitués de lentilles de galets, de graviers et de sables où s'observent des stratifications entrecroisées. Dans leur partie supérieure, ces dépôts sont formés de couches sablo-limoneuses, puis limono-argileuses correspondant à des dépôts de débordement et de fin de crue, dont certains peuvent servir de substrat à une pédogénèse (Wengler, 1993). Généralement, dans l'Holocène, ils se terminent par un sol peu évolué (Wengler et al., 1994); par contre, dans le Pléistocène supérieur, ces formations portent un calcarisol caractérisé par un profil calcaire différencié dans lequel se forment des croûtes calcaires (Ruellan, 1971 ; Pouget, 1980) de nature diverse qui, lors des périodes d'érosion, peuvent résister et permettre la conservation de l'ensemble de la séquence.

Dans l'oued Assaka, l'enregistrement sédimentaire et pédologique est différent. On y retrouve bien 3 phases sédimentaires principales, mais elles présentent des épaisseurs et des traits originaux qui seront décrits ultérieurement. On notera seulement l'important développement des phases limoneuses, qui ennoient tous les fonds de vallée préexistants, et constitue une caractéristique majeure de cette formation aisément repérable dans le paysage.

Les rapports entre cette accumulation majeure et d'autres formations quaternaires plus anciennes ou contemporaines sont quasiment inexistants, sauf au niveau de son exutoire, au débouché de Foum Assaka.
Dans cette zone, des lambeaux de nappe de galets d'origine alluviale et de tuf travertineux, attribuables à la partie inférieure de la terrasse alluviale, sont interstratifiés avec un niveau de plage marine à galets contenant des Thaïs haemastoma et des restes de coquilles appartenant à des Lamellibranches marins. Les restes de plage ancienne situés à $+2 \mathrm{~m}$ par rapport au niveau actuel de l'océan marquent les hauts stages du niveau marin de l'Ouljien (dont la partie la plus ancienne est contemporaine de l'Eémien dans le Nord de l'Europe) daté par U/Th entre 120000 ans et 60000 ans B.P. (Weisrock, 1980 ; Weisrock et al., 1999). Le dépôt alluvial précédemment décrit peut se corréler avec la partie inférieure limoneuse de la terrasse alluviale principale bien représentée une centaine de mètres en amont, dès l'entrée des gorges. Plus en amont, au niveau de Sidi Messaoud et de Bou Jerif, les datations en cours par la méthode U/Th de tufs travertineux situés dans la partie inférieure de cette terrasse, stratigraphiquement un peu plus récente que les niveaux de foum Assaka, indiquent une appartenance au stade isotopique 5 , tandis que plusieurs niveaux sédimentaires contiennent des artefacts moustériens. La partie supérieure de cette formation contient des chenaux plurimétriques dont le remplissage a livré des charbons de bois flottés datés par le radiocarbone à $27350 \pm 700$ ans BP (Gif-11650) et $27750 \pm 600$ ans BP (Gif-1 1648). Ces âges sont parfaitement compatibles avec ceux obtenus par la méthode de luminescence stimulée optiquement (OSL) appliquée à des grains de quartz extraits des mêmes dépôts sédimentaires : $27400 \pm 2600$ et $30200 \pm$ 2900 ans (N. Mercier-LSCE).

L'ensemble de ces observations et datations montre, en première approximation, que cette formation est attribuable à la plus grande partie du Soltanien de la chronologie marocaine (équivalent du Würm). Sa base en rapport avec le haut niveau marin de l'Ouljien peut être un peu plus ancienne et contemporaine de la fin de l'inter Tensiftien-Soltanien. Cela situe son âge entre les stades isotopiques 2 et 5 .

\section{3 - LES FORMATIONS RÉCENTES}

La formation alluviale principale est ensuite incisée sur une faible épaisseur dans sa partie terminale. Dans cette entaille se met en place un remplissage limoneux et travertineux de chenal dont un témoin a été conservé en aval du village d'Assaka. Des datations au carbone 14 situent ces dépôts entre $11880 \pm 100$ ans B.P. (Gif11022 ) sur coquille de Melanopsis sp. et $9965 \pm 100$ ans B.P. (Gif-11023) sur fragments d'œuf d'autruche. Ils constituent la première phase de remblaiement des vallées qui marque le début de l'Holocène au Maroc et correspond au Rharbien ancien (= Holocène ancien) de la chronologie du Quaternaire marocain (Wengler et al., 1994). L'incision qui a précédé leur mise en place s'est donc produite au cours du Tardi-Soltanien.

Ces phases d'incision et de remblaiement ont très peu marqué le paysage, puisqu'en amont de fort Bou Jerif, une butte témoin de la terrasse alluviale principale, située au milieu de la vallée et surplombant le lit mineur 
actuel (fig. 2), est couronnée par un glacis d'accumulation qui contient les vestiges d'un petit site épipaléolithique daté à partir de fragments de test d'œuf d'autruche à $7820 \pm 80$ ans B.P. (Gif-1 1643). A cette époque, ce lambeau de glacis était encore rattaché au versant et le paysage de bad-lands actuel n'existait pas.

Durant le Rharbien ancien, le paysage était celui d'une large plaine alluviale, comblée par les sédiments limoneux soltaniens, sur laquelle coulait l'oued Assaka dans une entaille peu profonde et relativement large, qui s'est peu à peu remplie de sédiments meubles sur une faible épaisseur, ne laissant à l'oued que des chenaux peu profonds qui facilitaient ses débordements lors des crues. Puis, à la suite d'un changement climatique, la mobilisation des versants a favorisé la mise en place d'un glacis d'accumulation entre environ 8000 et 7500 ans B.P. Période qui est probablement contemporaine du début de la phase d'incision majeure qui a recoupé les dépôts du Rharbien ancien, ainsi que toute la terrasse alluviale principale sur plus de vingt cinq mètres de hauteur jusqu'au niveau de la nappe de galets basale. Cette phase, bien connue dans d'autres régions (Weisrock et al., 1991 ; Wengler et al., 1994, Wengler, 1995), se situe entre les dépôts du Rharbien ancien et ceux du Rharbien moyen (= Holocène moyen).

Dans cette nouvelle gouttière large de quelques dizaines de mètres, une autre terrasse a été édifiée par l'oued. Cette basse terrasse atteint une épaisseur d'environ $6 \mathrm{~m}$ dans la zone de Bou Jerif, où elle est bien conservée. Elle débute par une charge de fond grossière, dont l'épaisseur dépasse deux mètres. On y observe des lentilles de galets, de graviers et de limon sableux jaune rougeâtre qui incorporent des blocs subanguleux de taille métrique (dropstones provenant des corniches proches). Ces éléments permettent de se faire une idée de la forte compétence de l'oued en crue. De nombreuses coquilles de gastéropodes sont présentes et un foyer y a été découvert. Les charbons recueillis sont en cours de détermination et de datation. La série se poursuit par des sables limoneux et des limons sableux qui témoignent d'une baisse de compétence généralisée. Ces sédiments ont été secondairement bioturbés. Ils contiennent des coquilles d'Otala sp. et montrent des carbonatations diffuses correspondant à des traits pédogénétiques. La présence de halite diffuse est constante. La séquence se termine par des limons à structure lamellaire correspondant à des sédiments de fin de crue déposés en milieu calme. Cette formation en cours de datation est semblable à la basse terrasse de la plus grande majorité des oueds maghrébins où elle correspond à l'optimum climatique du Rharbien moyen.

A Sidi Messaoud, en aval, la séquence est à peu de chose près semblable. Elle est toutefois moins épaisse et sa partie supérieure a été érodée, puis recouverte récemment par des limons de débordement. Deux foyers non construits étaient intercalés dans ces limons finement lités, dont l'épaisseur totale est d'ordre métrique. Le plus haut dans la stratigraphie a fourni un âge de $280 \pm 30$ ans BP (Gif-11522), qui indique un dépôt contemporain des hivers froids européens de la fin du XVII ${ }^{\text {èe }}$ début du
$\mathrm{XVIII}^{\mathrm{m} m e}$ siècle. Durant ces périodes, des dépressions sont centrées sur l'ouest de la péninsule ibérique avec un anticyclone sur l'Europe. Un dédoublement du flux perturbé en altitude, dont une branche atteint le Maghreb avec des coulées froides, a pu provoquer des pulsations humides éphémères à cette latitude, comme on le constate actuellement sur les situations synoptiques provoquant des précipitations dans cette région.

Actuellement, la formation du Rharbien moyen, ainsi que les dépôts plus récents qu'elle porte, sont totalement recoupés par l'érosion de l'oued Assaka et de ses affluents. L'incision linéaire, après avoir érodé la formation basale consolidée du Soltanien, attaque localement le substratum schisto-gréseux, notamment aux endroits qui forment des seuils où les eaux s'écoulent en cascatelles.

\section{3 - LES UNITÉS MORPHO-SEDIMENTAIRES DE LA FORMATION SOLTANIENNE}

L'enfoncement du lit de l'oued Assaka et de ses affluents a ouvert des coupes dans les dépôts du Soltanien, ce qui facilite leur étude de détail, notamment grâce au réseau de ravines transformant en bad-lands la majeure partie de la formation.

\section{1 - L'UNITÉ 1 BASALE}

C'est dans une vaste entaille formée à la faveur des failles qui affectent les terrains paléozoïques que l'oued Assaka a commencé à déposer des alluvions au début du Soltanien ou durant l'inter Tensiftien-Soltanien (équivalent de l'Eémien). Ces dépôts, dont la base ravinante repose directement sur le substratum primaire affecté d'un fort pendage, sont constitués par une nappe de galets formée d'un ensemble de lentilles emboîtées les unes dans les autres. Chacune d'entre elles contient des éléments très roulés (graviers et galets de dimension décimétrique à métrique) disséminés dans une matrice limono-sableuse carbonatée présentant une porosité racinaire et des caractères hydromorphes. L'étude des variations latérales de faciès montre que dans l'axe de la vallée, les dimensions des éléments grossiers sont généralement plus importantes que vers les rives.

L'épaisseur de cette unité n'excède pas 2 à $3 \mathrm{~m}$, sauf dans les zones de surcreusement du substratum. Les affleurements de Sidi Messaoud démontrent qu'elle occupe la plus grande partie du fond de la vallée lors de sa mise en place. Elle se termine par une diminution rapide du nombre et de l'importance des lentilles de graviers et de sables au profit de la matrice à dominante limoneuse qui prend de plus en plus d'importance. Globalement, la proportion et la taille des éléments grossiers diminue également vers le sommet.

\section{2 - L'UNITÉ 2 PRINCIPALE}

C'est par un passage progressif sur quelques dizaines de centimètres d'épaisseur que l'on passe à une série limoneuse comportant des traces de colonisation racinai- 


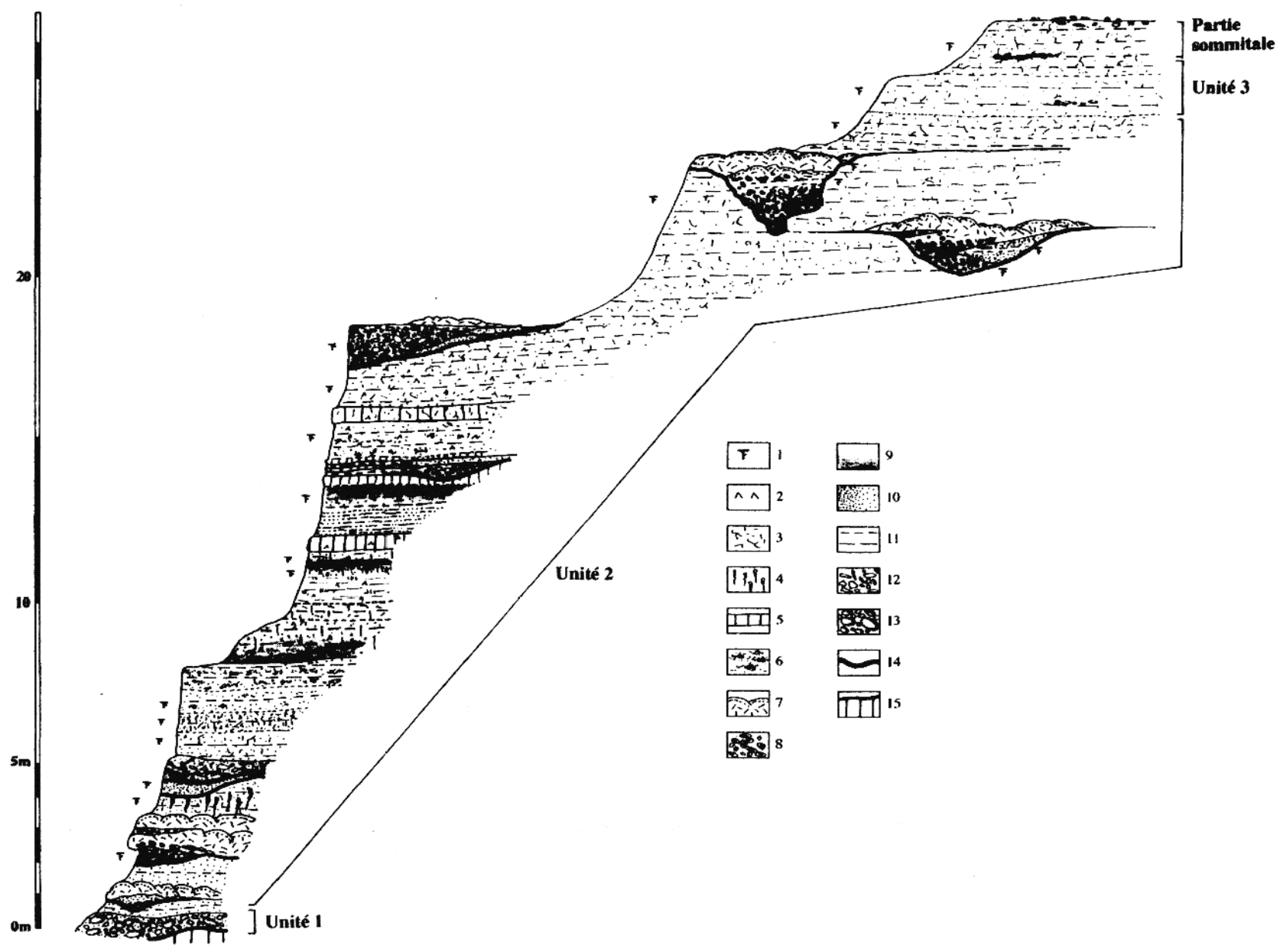

Fig. 4 : Stratigraphie de la terrasse soltanienne en aval de Bou Jerif.

1 : malacofaune. 2 : sel (halite, gypse). $3:$ traces de racines. $4:$ taches d'oxydo-réduction. $5:$ niveau calcaire. $6:$ concrétions carbonatées. $7:$ tuf travertineux. $8:$ oncolites. $9:$ niveau gris hydromorphe. $10:$ sable. $11:$ limon et argile. $12:$ cailloux anguleux. 13 : galets. 14 : contact érosif. $15:$ grés fins verts et silstone argileux cambriens.

Fig. 4 : Stratigraphy of the Soltanian terrace downstream Bou Jerif.

1 : malacofauna. $2:$ salt (halite, gypsum). $3:$ root marks. $4:$ oxidation-reduction spots. $5:$ calcareous level. $6:$ calcareous concretions. $7:$ travertine tufa. 8 : oncolites. $9:$ grey hydromorphic level. $10:$ sand. $11:$ silt and clay. $12:$ angular pebble $13:$ pebble. 14 : erosion surface. 15 : Cambrian green fine-grained sandstone and argillaceous siltstone.

re et des traits hydromorphes postsédimentaires. Ceux-ci sont à mettre en relation avec les mouvements de battement de la nappe phréatique qui ont provoqué des phénomènes d'oxydo-réduction et l'induration de la nappe de galets, ainsi que des 2 à 3 premiers mètres de limons par des précipitations de carbonates. Ces limons proviennent de fins de crues lors des débordements de l'oued Assaka, qui occupe durant cette période des chenaux de quelques mètres à plus d'une dizaine de mètres de largeur.

Cette unité est la plus épaisse de toute la formation. Dans la zone de Bou Jerif (fig. 4), elle atteint 27-28 m d'épaisseur et dans celle de Sidi Messaoud $35 \mathrm{~m}$ (fig. 5). On y rencontre deux types de faciès principaux qui, selon leur répartition spatiale, expriment des passages latéraux entre les chenaux de l'oued et leurs rives. Des niveaux limoneux, plus ou moins sableux, se déposent sur celles-ci, tandis que l'oued, au hasard de ses crues et de leurs compétences, creuse plus ou moins profondé- ment les niveaux sous-jacents et y forme des chenaux ravinants, qui périodiquement se comblent.

Ces chenaux présentent des séquences de remplissage particulières. Ils se comblent peu à peu de graviers avec des lentilles de sable particulièrement développées sur leurs bords, puis l'hydrodynamisme diminue et des oncolites se forment autour de fragments de roseaux, de charophytes et de Melanopsis sp. Enfin, dans un milieu encore plus calme, un premier tuf travertineux s'édifie. Il est constitué de lamines stromatolithiques construites par des cyanobactéries, qui forment des boules aplaties à noyau complexe, souvent de nature oncolitique. Ces boules de dimension pluridécimétrique à métrique sont jointives et donnent à cette construction un aspect superficiel mamelonné. Ces édifices travertineux ne se développent pas uniquement dans l'axe du chenal, mais aussi le long des rives directement au contact des limons de débordement. Ils forment des édifices de plusieurs dizaines de mètres de longueur, dont l'épaisseur dépasse 
parfois le mètre avec des superpositions de plusieurs phases de construction séparées par des épisodes de destruction partielle et de remaniement des édifices précédents.

Cette séquence sédimentaire constitue un cyclothème de base qui s'exprime avec diverses variantes dans les chenaux recoupés par l'érosion à différents niveaux de cette unité. Ces chenaux s'emboîtent les uns dans les autres avec des remplissages plus complexes où plusieurs cyclothèmes, parfois incomplets, se superposent. Dans le quart inférieur de cette unité, un niveau continu de chenaux peu profonds est remarquable. Il est le seul à présenter un remplissage uniquement formé d'éléments schisteux peu roulés issus des versants. Il met en relief l'importance exceptionnelle des apports latéraux locaux dans l'alimentation de l'oued. Associés à ces faciès de chenaux, se développent également des niveaux hydromorphes grisâtres et plus ou moins carbonatés où la malacofaune est abondante. Ils indiquent un milieu de dépôt anoxique, riche en matière organique, formé sous l'eau. Un tel milieu s'observe aujourd'hui encore dans les gueltas qui s'égrènent le long du lit de l'oued Assaka, et le long du chenal, dans les portions à eaux dormantes ou à écoulement laminaire durant l'étiage.

Les faciès de bordure, qui occupent la majorité du lit majeur et envahissent les lits des oueds affluents, s'organisent en couches horizontales de texture souvent voisine, limoneuse plus ou moins sableuse, où seuls s'individualisent bien les niveaux sableux grisâtres qui annoncent le passage latéral à un chenal. L'épaisseur de ces divers niveaux varie de quelques centimètres à quelques dizaines de centimètres. Leurs limites sont souvent diffuses et leur couleur est généralement beige avec parfois une dominante grise. (7,5 YR 4,5 à 7/3 à $6)$. Ils proviennent des apports longitudinaux issus de l'amont de l'oued et contiennent dans la partie inférieure de cette unité à Sidi Messaoud quatre niveaux d'incendie que l'on peut suivre sur quelques dizaines de mètres. Ils sont marqués sur le terrain par un liseré centimétrique rubéfié surmonté d'un lit charbonneux. Par ailleurs dans cette unité, on constate la présence régulière de halite et de gypse, soit cristallisé dans les pores, soit sous forme diffuse. Ceci s'accorde avec le caractère légèrement salé des eaux de l'oued et une concentration des sels par remontée capillaire et évaporation dans les sédiments, peu après leur dépôt. Dans les zones de contact avec les chenaux se développent des phénomènes de gleyification localisés sur quelques dizaines de centimètres. Certaines couches présentent une évolution post-sédimentaire avec des traits pédologiques (traces de racines, structure polyédrique, traits carbonatés discontinus...) et contiennent des restes de grande faune, de malacofaune et des industries préhistoriques.

\section{3 - L'UNITÉ 3 SUPÉRIEURE}

Elle est formée suivant les lieux par un ensemble de une à quelques couches qui reposent en concordance sur l'unité 2 . Son épaisseur varie entre 1 et quelques mètres selon les endroits et elle est toujours plus développée au niveau des confluences des oueds affluents. Elle présente la caractéristique d'être constituée de sédiments limono-sableux à limono-argileux de couleur ocre à dominante rouge à brunâtre (5 YR 4/6). Leur fraction sableuse comporte une proportion importante de grains picotés anguleux (40 à 60\%) témoignant d'une activité éolienne locale importante et de la reprise des sables éolisés par les eaux courantes. Les indices d'un transport éolien plus important sont par contre plus faibles. La structure de ces couches dépend de leur texture et oscille entre la structure granulaire et polyédrique. Des traits carbonatés et hydromorphes sont localement présents et liés à des pédogenèses peu évoluées.

Ces sédiments rubéfiés sont très bien représentés dans les formations alluviales des oueds affluents issus des chaînons anti-atlasiques que l'oued Assaka traverse dans la dernière partie de son cours. Ils contiennent de la palygorskite et proviennent du démantèlement d'anciens sols rouges développés sur les roches paléozoïques qui forment les versants des reliefs. La présence de ces sédiments rubéfiés dans la partie supérieure de la formation soltanienne de l'oued Assaka indique que durant la période postérieure à 27000 ans BP, cette zone du bassin versant a fourni, sous l'action de pluies localisées, une part importante du débit solide qui n'a pu être totalement évacué vers l'aval.

\section{4 - PARTIE SOMMITALE}

La formation se termine selon les endroits par une ou deux couches ocre rouge (5YR 5,5/6) sablo-limoneuses à limono-argilo-sableuses riches en plaquettes schisteuses aux arêtes non émoussées. Leur épaisseur est généralement inférieure au mètre et elles contiennent de nombreux gastéropodes terrestres. Les traces de racines y sont fréquentes et dans les faciès relativement argileux, une structure polyédrique se développe, tandis que dans les faciès plus sableux, elle est plutôt à tendance granulaire. L'analyse sédimentologique y révèle la présence de sables éolisés remaniés.

Cette unité correspond à des colluvions plus ou moins vannées par le vent. Formées sur les versants schisteux paléozoïques, elles ont été mobilisées vers les fonds de vallées, où elles constituent un glacis d'accumulation. Sur les bords actuels des gorges de l'oued Assaka, la racine de ce glacis est encore visible et il continue de fonctionner tout en étant peu productif.

Si le début du fonctionnement de ce glacis est peutêtre contemporain de la fin du Soltanien, l'essentiel de son histoire date du Rharbien, car un stationnement des hommes à l'Epipaléolithique se trouve scellé à l'intérieur de ses sédiments. Le glacis est arrivé à son maximum d'extension en atteignant le centre de la vallée durant la phase aride qui sépare le Rharbien ancien du Rharbien moyen, puis il a été en grande partie disséqué par l'érosion.

Cette partie sommitale ne fait donc pas partie de la formation soltanienne, elle vient seulement la recouvrir. 


\begin{tabular}{|l|c|c|c|c|}
\hline \multicolumn{2}{|c|}{ Taxon } & Sidi Messaoud & Bou Jerif & Total \\
\hline Bovini & Bos primigenius & 17 2(ra+n2) & 6 & 25 \\
\hline Alcelaphini & aff. Connochaetes sp. & 1 & 2 & 3 \\
\hline cf Antilopini & & 2 & & 2 \\
\hline cf Reduncini & & 1 & & 1 \\
\hline Bovidé indéterminé & taille moyenne & 2 & $1(\mathrm{cg})$ & 3 \\
\hline Equidés & Equus cf. asinus & 2 & 6 & 8 \\
\hline Camélidés & Camelus aff. thomasi & 2 & & 2 \\
\hline Rhinocérotidés & & 1 & & 1 \\
\hline Mammifère indét. & & 1 & & 1 \\
\hline Aves & & $1(2 e ́ \mathrm{ng})$ & & 1 \\
\hline \multicolumn{3}{|l}{} \\
\hline
\end{tabular}

Tab. 1: Liste faunique des grands mammifères et nombre de restes déterminés dans l'oued Assaka entre 1999 et 2001. Tab. I : Large mammals fauna distribution and number of determined remains in the Wadi Assaka since 1999 to 2001.

\section{4 - FAUNE ET ENVIRONNEMENT}

Le contenu paléontologique des différentes couches de la formation soltanienne est directement observable, grâce au décapage naturel des niveaux par l'érosion et aux nombreuses ravines qui les recoupent. Celui-ci comprend de la malacofaune, de rares éléments de micromammifêres et des restes de grands mammifères qui sont souvent réduits à l'état de fragments sous l'action du sel et de la dessiccation dès qu'ils séjournent à l'air libre. Des études préliminaires ont été conduites dans deux endroits privilégiés : la confluence des oueds Bou Smara et Assaka au niveau de Sidi Messaoud et, plus en amont, dans la zone de Bou Jerif.

Les observations concernant la faune mammalienne (tab. 1) portent sur une quarantaine de pièces qui proviennent de plusieurs niveaux de l'unité 2 . A Bou Jerif, 6 niveaux répartis sur les deux tiers supérieurs de l'unité en question ont livré des restes relativement peu nombreux. Il s'agit soit de dents (en séries mandibulaires ou isolées), soit d'éléments du squelette appendiculaire. Ces restes appartiennent pour l'essentiel à des Bovini et des Equidés, ainsi qu'à des Alcelaphini et Antilopini.

Dans le site de Sidi Messaoud, 7 couches réparties sur toute l'unité 2 , épaisse de $35 \mathrm{~m}$, unt livré des restes de faune. Elles ont fourni 31 pièces déterminables. La majorité de celles-ci proviennent de la couche 18 dans laquelle sont présents, au même niveau, des artefacts moustériens. Le taxon le plus représenté est Bos primigenius. Il est associé à un autre bovidé de taille moyenne, à des Alcelaphini comme Connochaetes sp., à l'âne, ainsi qu'à des Antilopini et Reduncini. Il faut également noter la présence de Rhinocérotidés et d'un Camélidé (Camelus aff. thomasi), qui n'était connu jusqu'à présent que dans l'Holocène.

Sur le plan taphonomique, il faut remarquer que certains ossements étaient en connexion anatomique, ce qui suppose un faible transport et un enfouissement rapide des parties de carcasses suite à des débordements probablement saisonniers de l'oued qui ont entraîné un dépôt de limons.
Cette première approche ne fournit que des indications globales, mais elle indique que la vallée de l'oued Assaka était fréquentée par de grands herbivores qui ont besoin d'une biomasse suffisante pour se nourrir. Le milieu dans lequel vivaient ces animaux présentait une strate herbacée parsemée de buissons beaucoup plus développée que celle observable aujourd'hui. Elle est actuellement limitée à quelques espaces plus humides dans les ravines qui permettent d'accéder au lit de l'oued et comporte principalement des épineux (Launea $s p$.) très clairsemés. Le lit mineur est en partie occupé par des lauriers roses (Nerium oleander), des tamaris (Tamarix sp.), des peupliers (Populus sp.), des roselières et des espaces herbacés. Dans les oueds affluents servant de zone refuge, quelques arganiers (Argania spinosa) résistent, profitant des sous-écoulements. Les bordures limoneuses du lit majeur sont par contre dénudées, tandis que les versants et les collines voisines sont le domaine d'une steppe à Euphorbiacées (Euphorbia echinus, E. Regis Jubae et Senecio Anteuphorbium) (J.P. Peltier, 1982). Elle ne subsiste dans ce bioclimat aride à la limite du désertique (précipitations moyennes annuelles : $100 \mathrm{~mm}$ ), que grâce aux précipitations latérales apportées par les advections d'air maritime sous la forme de brouillards côtiers ou de stratus bas, qui pénètrent d'une vingtaine de kilomètres à l'intérieur des terres. Ce milieu ne paraît pas correspondre à celui dans lequel vivaient les mammifères au moment du dépôt de la terrasse soltanienne, qui nécessite un couvert végétal plus dense favorisant la vie de grands herbivores.

Les sédiments de la terrasse soltanienne sont très pauvres en microfaune. Seule une dent (M2d) de Gerbillus cf campestris a été identifiée dans un chenal de la partie supérieure de l'unité 2 à Bou Jerif. Par contre, le niveau de glacis daté à 7820 ans BP au niveau du site épipaléolithique a livré une vingtaine de dents de Meriones cf lybicus. Ce taxon préfere les biotopes steppiques à végétaux buissonnants et il est particulièrement abondant dans les lits d'oued (Aulagnier et Thévenot, 1986). Bien que la faible diversité spécifique rencontrée dans les échantillons fossiles ne permette pas, pour l'instant, d'envisager une interprétation paléoenvironnementale, il faut remarquer que ces 
espèces sont bien adaptées à un climat aride. Un échantillon de microfaune actuelle trouvé sur un placage contre la paroi d'une ravine à Sidi Messaoud fournit une référence récente. Trois rongeurs (Gerbillus cf. campestris, Meriones sp. et Eliomys quercinus), un macroscelidé (Elephantulus rozeti) et un insectivore (Crocidura sp.) ont été reconnus. Ces restes proviennent de pelotes de régurgitation de rapaces qui restent, pour l'essentiel, à l'origine des petits ossements trouvés dans les sédiments. Cette association indiquant un milieu rocailleux, steppique, elle rend assez bien compte du milieu environnant.

Les mollusques continentaux sont de loin les plus abondants et les mieux conservés dans cette formation. Les types d'assemblages récoltés dans l'unité 2 sont très variés en ce qui concerne leur richesse et leur composition spécifique. La coupe de "Tour aval" à Bou Jerif, plus marginale par rapport à l'axe de l'oued, comporte une majorité d'assemblages strictement terrestres, à l'exception de rares niveaux travertineux. Dans la séquence de Sidi Messaoud, au contraire, la composante aquatique n'est jamais absente.

Un premier examen de trente cinq échantillons révèle quatre grands types d'habitats terrestres, dont certains ne paraissent pas avoir d'analogues actuels :

- prairies humides caractérisées par des assemblages quasi mono-spécifiques à Cochlicella barbara ;

- milieux steppiques, à faible biomasse végétale, caractérisé par des assemblages riches en Helicopsis sp., Ferussacia sp. et Truncatellina sp. ;

- milieux de type "matorral", avec Rumina decollata et Otala spp. ;

- habitats mésophiles, à biomasse végétale plus abondante et à structure plus complexe, caractérisés par des assemblages abondants et diversifiés à Otala spp., Truncatellina sp., Vitrea sp., Vallonia sp., Ferussacia sp., "Helix calpeana"...

Lorsqu'elle est présente, la composante aquatique des assemblages témoigne de conditions hydrochimiques et hydrodynamiques très variées :

- marécages et mares temporaires plus ou moins saumâtres à Pseudamnicola sp.;

- assemblages à Melanopsis, Pseudamnicola et Bythiospeum témoignant d'eaux plus courantes ;

- assemblages plus diversifiés à Ancylus fluviatilis, Gyraulus crista, Pisidium sp., Bythiospeum, Melanopsis etc., témoignant de milieux oligohalins (3,5\%o au maximum) riches en végétation aquatique.

Il semble que ces assemblages soltaniens soient très originaux et n'aient pas de stricts analogues actuels. P. Jodot avait d'ailleurs remarqué, dès 1952, l'originalité morphologique des Melanopsis des dépôts soltaniens de l'oued Assaka qu'il avait décrits sous le nom de "Melanopsis arbalensis PEREZ forme assakaensis" (Jodot, 1965). L'analyse détaillée de ces séquences devrait permettre, à terme, de caractériser à la fois l'évolution des environnements terrestres et aquatiques au fur et à mesure de la sédimentation. Elle devrait aider, en particulier, à comprendre le cadre paléoécologique qui a permis le développement des formations travertineuses.

\section{5 - INTERPRÉTATION ET DISCUSSION}

L'analyse de cette terrasse alluviale principale met en relief la diversité des faciès rencontrés qui, outre les variations latérales internes à une même phase sédimentaire, souligne les changements qui sont intervenus dans la dynamique fluviale consécutivement aux modifications du climat.

Suite à un creusement majeur, l'unité 1 marque une première phase d'aggradation contemporaine de la régression fini-ouljienne. Sa composition sédimentologique indique que l'oued avait épisodiquement des crues très violentes occupant toute la vallée. Elles permettaient d'évacuer une grande partie des sédiments vers l'océan. Ce n'est qu'en fin de crue que les éléments grossiers formaient des lentilles emboîtées présentant des stratifications obliques qui se déposaient rapidement sur toute la largeur du lit (environ $200 \mathrm{~m}$ ) avec quelques variations granulométriques vers les rives. Cela suppose des pluies importantes sur l'ensemble du bassin versant durant des périodes courtes susceptibles d'impliquer un régime torrentiel. Régime qui s'amortit rapidement au cours du temps, entraînant une diminution de la dimension des éléments grossiers dans les dernières lentilles.

La courte phase de transition entre l'unité 1 et 2 implique un changement rapide du régime pour des raisons diverses : changement de la répartition des pluies dans l'année, variation de la pluviométrie, développement de la végétation dans le bassin versant... Actuellement, les informations disponibles ne permettent pas de choisir entre ces hypothèses.

Ensuite, dans cette portion du cours, seuls se déposent des sédiments limoneux provenant du débordement d'un oued qui n'a plus la compétence de convoyer la charge de fond jusque dans sa partie distale. L'oued occupe des chenaux apparemment peu profonds et multiples, au fond desquels s'accumulent galets et graviers, ce qui marque encore une certaine irrégularité du régime. L'alimentation régulière de ces chenaux provoque la gleyification des limons encaissants. La baisse de compétence des eaux et le caractère pérenne de l'oued permettent, peu à peu, la sédimentation de sables et de limons, puis le développement de masses travertineuses, qui transforment le lit de l'oued en une succession de vasques ou gueltas dans lesquelles prolifère une faune particulière adaptée à des eaux légèrement salées. Actuellement, à Foum Assaka, dans un contexte lagunaire, des formations travertineuses jeunes s'édifient dans des eaux fortement chargées de matières en suspension. Elles peuvent constituer un modèle actuel intéressant.

Au cours de ce cycle de comblement des chenaux, des faciès anoxiques grisâtres à noirâtres se forment dans le lit et sur ses marges en fonction du débordement de l'oued. Lorsque le chenal s'avère en grande partie comblé, à la faveur de crues plus importantes et plus brutales, le lit de l'oued se déplace. Les eaux plus turbides incisent les niveaux limoneux sous-jacents, créant un nouveau chenal. Ensuite, s'instaurent peu à peu les conditions nécessaires à une nouvelle phase de comble- 


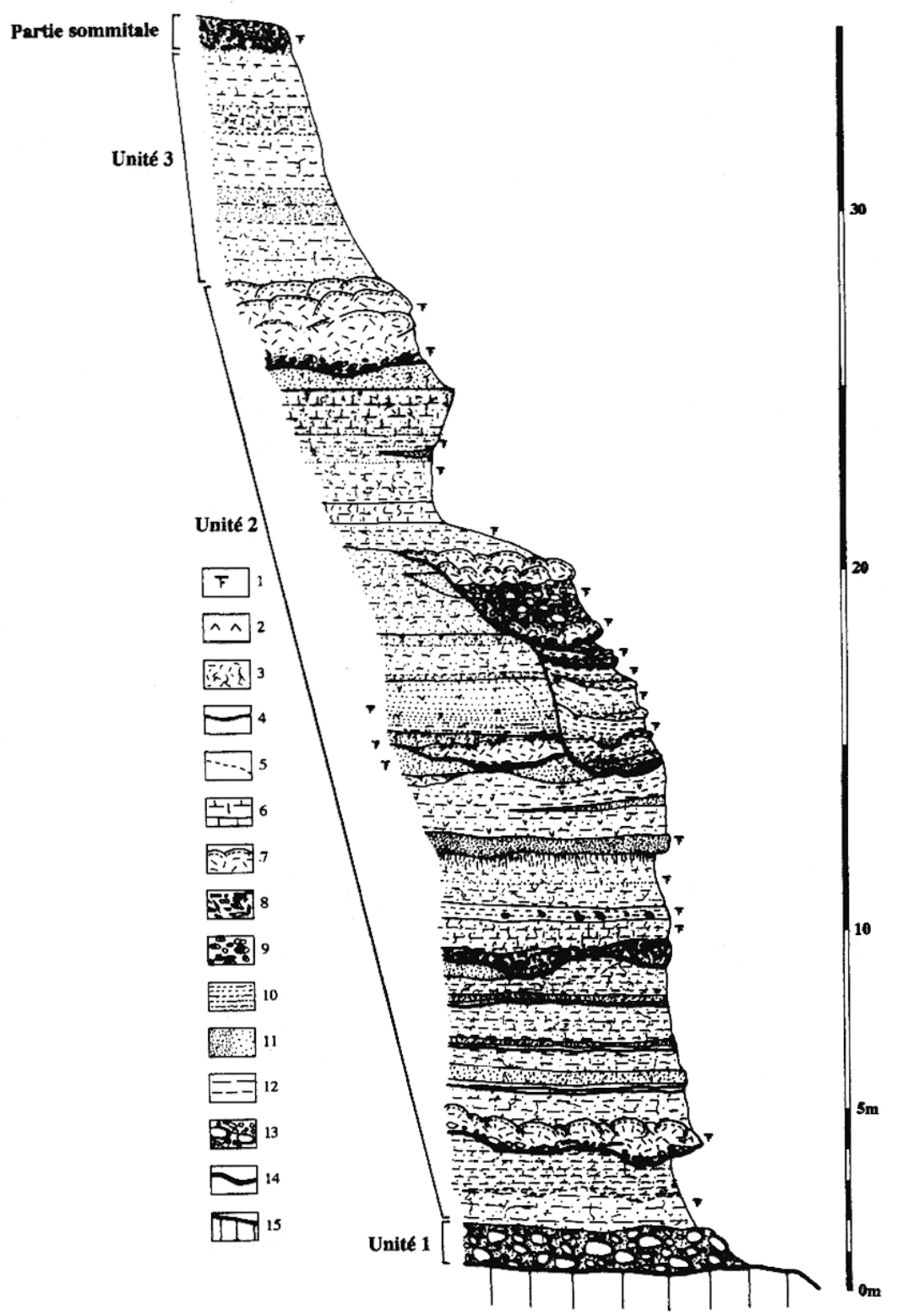

Fig. 5 : Stratigraphie de la terrasse soltanienne au niveau du Marabout de Sidi Messaoud.

$1:$ malacofaune. $2:$ sel (halite, gypse). $3:$ traces de racines. 4 : niveau d'incendie. 5 : limite de gleyification. 6 : niveau calcaire. 7 : tuf travertineux.

8 : cailloux anguleux. 9 : oncolites. $10:$ sables lités. $11:$ sable. $12:$ limon et argile. $13:$ galets. 14 : contact érosif. 15 : grés fins verts et silstone argileux cambriens.

Fig. 5 : Stratigraphy of the Soltanian terrace near the Marabout of Sidi Messaoud.

1 : malacofauna. 2 : salt (halite, gypsum). 3 : root marks. 4 : fire level. 5 : limit of gleying process. $6:$ calcareous level. $7:$ travertine tufa. $8:$ angular pebble. $9:$ oncolites. $10:$ bedded sand. 11 : sand. $12:$ silt and clay. $13:$ pebble. 14 : erosion surface. 15 : Cambrian green fine-grained sandstone and argillaceous siltstone ment, concomitante d'une baisse de la compétence des eaux fluviatiles. Ces variations de régime peuvent s'interpréter en termes de modifications climatiques à l'intérieur de la période soltanienne. Elles marquent une certaine rythmicité du climat où alternent des périodes de précipitations fortes et brutales à l'origine des phases d'incision et des périodes où des précipitations plus régulières alimentent l'oued sans que des crues trop brutales ne viennent perturber la construction des édifices travertineux. D'autre part, ces précipitations permettent de recharger les nappes phréatiques du karst anti-atlasique, qui sont à l'origine des sources et rendent pérenne l'oued Assaka dans la partie inférieure de son cours. Il est toutefois évident que les périodes de fortes précipitations sont sans commune mesure avec celles qui ont permis le creusement de la vallée et la mise en place de l'unité 1. Durant les phases d'alimentation régulière, l'oued sort périodiquement de son lit lors des crues permettant l'étalement de sables et de limons dans le lit majeur. Entre deux crues, ces sédiments sont colonisés par une végétation à l'origine d'une biomasse végétale plus ou moins importante selon les périodes. Ces végétaux laissent des traces dans les sédiments et favorisent selon les milieux le développement d'une malacofaune particulière. Ces milieux, certainement plus riches que l'actuel, attirent une faune de grands mammiferes herbivores, ainsi que des prédateurs, dont l'homme. Des sols peu évolués se mettent en place avec une pédofaune suffisamment active pour entraîner des remaniements à l'intérieur des couches et faire disparaître certaines limites de couche ; des carbonatations localisées peuvent également se développer.

Tout au long de cette unité, il est difficile de percevoir le rythme des cycles, car l'érosion ne recoupe pas tous les chenaux et en a détruit certains. Cependant, dans la partie inférieure, on note une certaine irrégularité, marquée d'une part par un épisode torrentiel, dû à des pluies importantes localisées sur les parties distales du bassin versant, qui a permis l'évacuation partielle d'apports de versant, et d'autre part par l'important dévelop- 
pement des phases limoneuses. Dans la partie supérieure, les cycles semblent se succéder à un rythme plus élevé, avec formation de chenaux secondaires qui s'emboîtent les uns dans les autres (fig. 5). Ils semblent traduire une certaine instabilité climatique qui reste à préciser. L'ensemble de cette unité, compte tenu des éléments paléontologiques recueillis et des différents milieux rencontrés, apparaît globalement plus humide que l'actuel, avec un oued pérenne, au moins au niveau des gueltas, et une large plaine alluviale limoneuse colonisée par une végétation variée, buissonneuse et herbacée. Les cycles décelables, compte tenu de leur nombre, peuvent être liés aux oscillations climatiques rapides bien identifiées dans les glaces du Groënland (carotte GRIP). Cependant, de nombreuses précisions paléoclimatiques et chronologiques manquent encore pour confronter les données de l'oued Assaka avec les oscillations de « Dansgaard-Oeschger » (Dansgaard et al., 1993) ou avec les événements de Heinrich (Heinrich, 1988), ainsi que pour comprendre le détail des relations possibles.

Dans l'unité 3, qui n'est conservée que sur les bordures et au niveau des oueds affluents, le régime des pluies paraît relativement contrasté avec la mobilisation d'éléments grossiers et le développement de niveaux carbonatés. La composition et la coloration rougeâtre des sédiments, en indiquant leur provenance très locale, marquent là encore un épisode pluvieux concentré sur la partie du bassin versant limitée aux gorges d'Assaka. La forte composante en éléments éolisés d'une partie des sédiments montre à l'opposé l'importance du vent dans un paysage soumis à la sécheresse, où la couverture végétale est réduite. Ces caractéristiques ne sont pas sans rappeler les conditions qui règnent au cours du stade isotopique 2 (Rognon et Coudé-Gaussen, 1996) et s'accordent avec un climat de type aride où les rares pluies sont violentes et localisées. C'est dans cette ambiance que le comblement de la vallée atteint son maximum.

Dès lors, la phase d'incision qui suit est à mettre en rapport avec une période très aride à la pluviométrie très contrastée, tandis que la formation attribuable au Rharbien ancien marque un retour à des conditions plus humides avec des pluies mieux réparties favorisant l'installation d'une tendance biostasique. Celle-ci intervient au cours de la remontée des températures qui se situe entre le Dryas récent et le début de l'Holocène entre 12 000 ans et 8500-9000 ans B.P. Cette remontée est bien marquée dans les diverses courbes du $\delta^{18} \mathrm{O}$ et notamment dans celle de la carotte GRIP du Groënland (Johnsen et al., 1997).

Ces conditions vont peu à peu se dégrader avec un retour vers une nouvelle phase plus aride à caractère rhéxistasique perceptible dans l'unité sommitale plus récente, où les versants dénudés et soumis à la fragmentation sont mobilisés par les eaux de ruissellement et forment un glacis d'accumulation, tandis que l'oued, dans ce secteur distal, commence à inciser les niveaux précédemment construits et que l'action du vent sur les sédiments se renforce, accentuant le caractère aride du milieu. Compte tenu de l'imprécision des datations que l'on peut attribuer à ces phénomènes, il semble plausible de les mettre en rapport avec l'événement climatique abrupt de 8200 ans B.P. qui se traduit par un brusque refroidissement de l'ordre de $6^{\circ} \mathrm{C}$ en 50 ans, suivi d'une période équivalente pour revenir à la température initiale (Alley et al., 1997). Ce refroidissement a pu entraîner des précipitations importantes non régulières au niveau des latitudes qui nous intéressent et provoquer une reprise de l'érosion avec une mobilisation des formations superficielles sur les versants.

Un peu plus récemment, et après une période de transition marquée encore par des écoulements torrentiels qui laissent en place des lentilles de galets et de graviers dans le lit mineur, ainsi que des blocs partiellement roulés, éboulés des falaises qui bordent certaines parties des gorges de l'oued Assaka, des conditions humides se réinstallent. Elles favorisent le développement d'une tendance biostasique qui permet, cependant, la mobilisation des sédiments fins et leur dépôt sous forme d'une terrasse alluviale. Cette formation, d'une ampleur beaucoup plus faible que les dépôts soltaniens, est parfaitement connue dans différentes régions du Maghreb au Rharbien moyen (Wengler, 1993 ; Wengler et al., 1994).

Cette période de sédimentation est suivie d'un retour à des conditions plus rhéxistatiques, probablement accentuées par le rôle de l'homme dans la région, qui entraînent une reprise importante de l'érosion et l'incision des formations antérieures. Ces nouvelles conditions provoquent périodiquement des crues, dont les plus importantes laissent en bordure du lit des dépôts limonosableux de débordement qui se superposent aux formations rharbiennes antérieures, notamment au cours des $\mathrm{XVII}^{\mathrm{i} m e}$ et XVIII ${ }^{\mathrm{e} m e}$ siècle. Les conditions météorologiques de cette époque étant mieux connues, elles peuvent servir de modèle pour déchiffrer en partie celles du passé.

\section{6 - CONCLUSIONS}

Le système de terrasses alluviales de l'oued Assaka, avec trois formations aujourd'hui identifiées, $s$ 'avère très incomplet et cependant tout à fait remarquable par l'extraordinaire développement de sa terrasse intermédiaire. Celle-ci s'est construite entre 120000 et environ 24000 ans B.P. (hypothèse longue) ou entre 60000 et environ 24000 ans B.P. (hypothèse courte) durant une phase de régression marine. Elle couvre donc une grande partie du Soltanien, mais comme de nombreuses séries continentales, cette séquence fluviatile est discontinue. Au-delà des 3 phases sédimentaires principales, qui marquent des changements majeurs dans la dynamique de mise en place des sédiments, une recherche des grandes coupures chronologiques qui peuvent exister dans une telle formation est en cours de réalisation à partir d'une série de datations croisées (U/Th, ${ }^{14} \mathrm{C}$ et OSL).

Dans l'unité 1 , on passe rapidement d'un climat à régime de pluies très irrégulier favorisant des écoulements torrentiels à un régime moins contrasté ne permettant que l'évacuation partielle des sédiments fins et la formation d'importants dépôts limoneux constituant la 
majeure partie de l'unité 2. Ces dépôts contiennent des niveaux d'incendie et des apports latéraux, locaux, d'origine torrentielle, parfaitement préservés, qui sont la conséquence d'événements brefs. Par ailleurs, l'enfouissement rapide d'ossements de grands mammifères et des paléosurfaces occupées par les hommes du Paléolithique moyen montre que la sédimentation est un phénomène rapide dans les différentes parties de la vallée. Compte tenu de l'épaisseur des couches (plusieurs dizaines de centimètres), il est possible de considérer chacune d'entre elle comme un enregistrement sédimentaire à haute résolution susceptible de contenir des informations détaillées sur le milieu environnant qu'il devient nécessaire de rechercher.

Le dispositif morphologique de l'ensemble de la terrasse intermédiaire indique que l'aggradation majeure s'est effectuée dans cette vallée, aujourd'hui côtière, durant la dernière régression marine, tandis que le creusement vertical postérieur avait lieu en pleine phase de transgression (remontée marine postglaciaire). Cette contradiction totale avec le glacio-eustatisme confere à cette vallée et à son remplissage alluvial un caractère tout à fait exemplaire qui conduit à privilégier les facteurs d'explication climatiques (Blum et Törnqvist, 2000).

Les études paléontologiques réalisées jusqu'à présent montrent l'existence au sein de l'unité 2 d'une faune de grands mammifêres vivant dans une végétation plus fournie qu'à l'heure actuelle. Cela suppose un climat où les précipitations étaient plus abondantes et probablement plus régulières qu'aujourd'hui durant la mise en place de l'unité 2. Cette tendance climatique s'exprime également dans l'analyse des milieux de sédimentation qui montrent une grande diversité et mettent en relief des fluctuations climatiques mineures à caractère cyclique qui semblent disparaître avec l'augmentation de l'aridité marquée dans l'unité 3. Il serait, cependant, imprudent de conclure que le Soltanien dans son ensemble est une période globalement plus humide que l'actuel tant que la chronologie interne de la formation n'est pas connue précisément, car seules ont pu être enregistrées dans cette vallée les oscillations plus humides de cette période que l'on commence à mieux connaître. Il reste à prendre en considćration d'une part l'alimentation pérenne de l'oued Assaka par des sources et d'autre part le rôle de l'upwelling côtier responsable des brouillards qui entretiennent la végétation dans la zone côtière avant de se prononcer sur les caractéristiques climatiques du Soltanien dans cette région par rapport aux tendances climatiques globales. Autrement dit, les facteurs climatiques et morphodynamiques locaux (brouillards côtiers, pluies rares mais catastrophiques, configuration du bassin versant, pente de la basse vallée et du plateau continental, alimentation des nappes phréatiques, présence de gueltas) compliquent les enregistrements et surtout leur relation avec les mécanismes généraux tels qu'ils peuvent être connus dans l'Atlantique Nord entre les stades 5 et 2 (Grousset, 1998, 2001). Les données chronologiques, dont on dispose jusqu'à présent, n'autorisent pas à de tels rapprochements en l'état actuel des recherches.
Cette formation sédimentaire et l'enregistrement climatique qu'elle est susceptible de livrer apportent des faits nouveaux dans la mesure où les terrasses alluviales soltaniennes étudiées jusqu'à présent au Maroc et plus généralement au Maghreb ne représentaient que des portions de cette période. Elles n'enregistraient que les phases humides et les phases intermédiaires de passage aride-humide et humide-aride (Wengler et Vernet, 1992 ; Wengler et al., 1992 ; Weisrock et al., 1994). Dans le cas de l'oued Assaka, nous sommes en présence d'une séquence plus longue et plus détaillée en relation avec un bas niveau marin qui devrait fournir de nombreuses informations sur cette période.

\section{REMERCIEMENTS}

Tous nos remerciements vont à $\mathrm{P}$. Rognon et à $\mathrm{S}$. Occhietti pour leurs judicieux conseils, ainsi qu'à la faculté des lettres et sciences humaines d'Agadir et aux responsables du programme Éclipse du CNRS qui ont soutenu ce projet.

\section{BIBLIOGRAPHIE}

ALLEY, R.B., MAYEWSKI, P.A., SOWERS, T., STUIVER, M., TAYLOR, K.C. and CLARK, P.U., 1997 - Holocene climatic instability : a promi-rent, widespread event 8200 yr ago. Geology, $25,483-486$

ANDRES, W., 1977 - Studien zur jungquartären Reliefentwicklung des südwestlichen Anti-Atlas und seines saharischen Vorlandes, Marokko. Mainzer Geogr. Stud. 9, 148 p.

AIJIAGNIER, S. FT THEVENOT, M., 1986 - Catalogue des mammifères sauvages du Maroc. Trav. Inst. Chérifien, sér. Zoologie, 41, $146 \mathrm{p}$.

BALLAIS, J.-L., 1981 - Recherches géomorphologiques dans les Aurès (Algérie). Thèse de Doctorat ès Lettres, Paris I, 566 p.

BARATHON, J.-J., 1986 - Bassins littoraux du Rif oriental (Maroc) : évolution morphoclimatique et tectonique depuis le Néogène supérieur. Thèse de Doctorat ès Lettres, Poitiers, 547 p.

BHIRY, N., ROGNON, P. et OCCHIETTI S., 1989 - Origine et diagenèse des sédiments quaternaires de la vallée moyenne du Souss. Sciences Géologiques, Strasbourg, 84 (2), 139-148.

BHIRY, N., 1991 - Formations quaternaires de la vallée moyenne du Souss (Maroc). Thèse de Doctorat, Paris VI, 214 p.

BLUM, M.D. et TÖRNQVIST, T.E., 2000 - Fluvial responses to climate and sea-level change : a review and look forward. Sedimentology, 47 (suppl. 1), 2-48.

GROUSSET, F., 1998 - Les événements de Heinrich. Océanis, 24, 1, 5-22.

GROUSSET, F., 2001 - Les changements abrupts du climat depuis 60.000 ans. Quaternaire, 12, 4, 203-211.

HEINRICH, H., 1988 - Origin en conséquences of cyclic ice rafting in the northeast Atlantic Ocean during the past 130,000 years. Quaternary Research, 29, 142-152.

JODOT, P., 1965 - Malacologie continentale de quelques gisements quaternaires du littoral atlantique marocain. Notes Serv. Géol. Maroc, 25, 185, 101-114.

JOHNSEN, S.J., CLAUSEN, H.B., DANSGAARD, W., GUNDESTRUP, N.S., HAMMER, C.U., ANDERSEN, U., ANDERSEN, K.K., HVIDBERG, C.S., DAHL-JENSEN, D., STEFFENSEN, J.P., SHOJI, H., SVEINBJÖRNSDOTTIR, A.E., WHITE, J.V.C., JOUZEL, J. and FISHER, D., 1997 - The record along the Greenland Ice Core Project deep ice core and the problem of possible Eemian climatic instability. Journal of Geophysical Research, 102, 26.397-26.410. 
LEFEVRE, D., 1985 - Les formations plio-pléistocènes du bassin de Ksabi (Moyenne Moulouya, Maroc). Thèse de 3e Cycle, Bordeaux I, 265 p.

OLIVA, P., 1974 - Carte géomorphologique des Plaines de l'Anti-Atlas occidental au $1 / 100000^{\circ}$. Royaume du Maroc, Ministère des T.P. et des Comm., Direction de l'Hydraulique, Division des Ressources en eau.

PELTIER, J.-P., 1982 - La végétation du bassin versant de l'oued Souss, Maroc. Thèse Doctorat. ès-Sciences, Grenoble, 201 p, + vol. cartes et annexes.

POUGET, M., 1980 - Les relations sol-végétation dans les steppes sud-algéroises. Trav. et Doc. $\mathrm{n}^{\circ} 116$, O.R.S.T.O.M., Paris, 555 p.

ROGNON, P. et COUDE-GAUSSEN, G., 1996 - Changements dans les circulations atmosphérique et océanique à la latitude des Canaries et du Maroc entre les stades isotopiques 1 et 2 . Quaternaire, 7, n², 197-206.

ROGNON, P. et COUDE-GAUSSEN, G., 1996 - Palaeoclimates of Northwest Africa $\left(28^{\circ}-35^{\circ} \mathrm{N}\right)$ about 18,000 yr B.P., based on continental aeolian deposits. Quaternary research, 46, 118-126.

RUELLAN, A., 1971 - Les sols à profil calcaire différencié des plaines de la Basse Moulouya (Maroc oriental). Mémoire O.R.S.T.O.M. $\mathrm{n}^{\circ}$ 54, Paris, 302 p.

WEISROCK, A., 1980 - Géomorphologie et paléoenvironnements de l'Atlas atlantique (Maroc). Thèse de Doctorat ès Lettres, Paris $1,931 \mathrm{p}$.

WEISROCK, A., 1993 - Géomorphologie et paléoenvironnements de l'Atlas atlantique (Maroc). Notes et mémoires du Service géologique, $\mathrm{n}^{\circ} 332$. Editions du Service géologique du Maroc, Rabat, $487 \mathrm{p}$.

WEISROCK, A., OUAMMOU, A. et AïT HSSAiNe, A., 1991. - Erosion et sédimentation dans les oueds du Sud-Ouest marocain à l'Holocène. Physio-Géo., 22-23, 95-100.

WEISROCK, A., FONTUGNE, M. ET OUAMMOU, A., 1994 - Le Soltanien supérieur de l'oued Tamdroust (Province de Tiznit, Maroc atlantique). Revue de Géographie du Maroc, 16, 1-2, 351367.

WEISROCK, A, OCCHIETTI, S, HOANG, C.T, LAURIATRAGE, A., BREBION, P. et PICHET, P., 1999 - Les séquences littorales quaternaires de l'Atlas atlantique entre Agadir et Cap Rhir, Maroc. Quaternaire, 10, (2-3), 227-244.

WENGLER, L., 1993 - Cultures préhistoriques et formations quaternaires au Maroc oriental. Relations entre comportements et paléoenvironnements au Paléolithique moyen. Thèse de Doctorat d'Etat ès Sciences, Bordeaux I, 2 t, 1433 p.

WENGLER, L., 1995 - Dégradation ou modification du milieu au Maghreb durant l'Holocène : causes anthropiques ou naturelles? In : S. Van der Leeuw, "L'Homme et la dégradation de l'environnement". $\mathrm{XV}^{\mathrm{e}}$ Rencontres Internationales d'Archéologie et d'Histoire d'Antibes, 20-22 Oct. 1990, Juan-Les-Pins, Edit. APDCA, Juan-les-Pins, 315-329.

WENGLER, L. et VERNET, J.-L., 1992 - Vegetation, sedimentary deposits and climates during late Pleistocene and Holocene in Eastern Morocco. Palaeogeography, Palaeoclimatology, Palaeoecology, 94, 141-167.

WENGLER, L., VERNET, J.-L., BALLOUCHE, A., DAMBLON, F. et MICHEL, P., 1992 - Signification des paléomilieux, cultures préhistoriques et climats durant le Pléistocène récent et l'Holocène au Maroc oriental. In : J.-L. Vernet, "Les charbons de bois, les anciens écosystèmes et le rôle de l'homme". 10-13 Sept. 1991, Montpellier, Bull. Soc. Bot. de France, 139, 507-529.

WENGLER, L., VERNET, J.-L. et MICHEL, P., 1994 - Événements et chronologie de l'Holocène en milieu continental au Maghreb. Les données du Maroc oriental. In : "Échelles des variations chronoclima-tiques quaternaires et réponses des environnements". Coll.
INQUA-AFEQ Quaternaire 1, 15-18 Mars 1994, Montpellier, Quaternaire, 5, (3-4), 119-134.

ZARKI, A., 1999 - Evolution de la sédimentation fluviatile en Basse Moulouya (Maroc) au cours de l'Holocène. Thèse Univ. Tours, 370 p. 